\title{
Multiagent-Based Flexible Automation of Microproduction Systems Including Mobile Transport Robots
}

\author{
Holger Voos \\ University of Luxembourg \\ Automatic Control Research Group \\ rue R. Coudenhove-Kalergi, L-1359 Luxembourg \\ holger.voos@uni.lu
}

\author{
Suparchoek Wangmanaopituk \\ Sirindhorn Int. Institute of Technology \\ Thammasat University, Thailand \\ suparchoek_w@yahoo.com
}

\begin{abstract}
In microproduction, i.e. in the production and assembly of micro-scale components and products, fully automated systems hardly exist so far. Besides the requirements of handling small parts with extreme precision, small batch sizes of highly customized products are among the main challenges. Therefore, economic microproduction requires very flexible production systems with a high level of automation. This contribution proposes a new concept of such a system that provides two main innovations. First, the proposed concept integrates stationary production machines and mobile transport robots in order to configure rapidly changing production processes in real-time. Besides this distributed flexible system structure, also the overall automation system consisting of the Manufacturing Execution System (MES) and the shop floor control is designed in a distributed form as a multiagent system. This distributed automation system is especially suited to automate flexible production scheduling and resource allocation processes but also integrates the multi-robot transport system.
\end{abstract}

\section{Introduction}

Enabled by innovations in microelectronics, microsystems technologies, mechatronics and optical technologies, there is a dramatically increasing demand for precisely manufactured microcomponents or -products consisting of various materials. While considerable progress has been made in the single process steps of micromachining and -assembly in recent years, see e.g. [1], [2] for a comprehensive overview, so far no complete industrial microproduction systems exist where those process steps are connected to an automated overall production process. However, only such fully automated microproduction systems will offer a cost-efficient and fast production of microproducts in the future. In addition, one big challenge in microproduction is mass customization, i.e. the manufacturing of a large variety of microproducts in small quanti- ties that are highly adapted to special customer needs [3]. These requirements call for a highly flexible microproduction system that offers all necessary production and assembly processes while being able to connect and schedule these processes in a very flexible way in short time.

In state-of-the-art microproduction solutions (see [1], [3]), two different approaches can be distinguished. One approach comprises desktop factories, i.e. small scale factories for microproducts, see e.g. [3]. However, such desktop factories are not suitable for cutting, turning or milling processes, since these processes require highly dynamic machining with sufficient stiffness and damping for high precision [1]. Therefore, desktop factories are mainly used for microassembly only. In the second approach in microproduction, where also cutting, turning, milling or other machining processes are included, the related machines have dimensions as in macro production because of the necessary heavy machine tables, see e.g. Fig. 1 for two commercially available machines. A highly flexible microproduction system thus has to integrate macro-size machines and assembly units for the ultra-precise handling and processing of micro-scale parts and products. This contribution proposes a new concept of such a highly flexible microproduction system that fulfils these requirements and is based on two main innovations.

First, the concept integrates stationary macro-scale machines for microproduction and -assembly but also mobile transport and part-feeding robots in order to configure rapidly changing production processes in real-time. While stationary robotic manipulators are important components of manufacturing processes since decades, the application of mobile robots equipped with manipulators in industrial environments is still at a very early stage and mainly a topic of research. This is due to the very complex tasks that have to be fulfilled autonomously by the robot in a highly dynamic environment and the interaction with human co-workers [4], [5]. First applied solutions can be found in the area of automated guided transport vehicles (AGVs) in manufacturing, see e.g. [4], [12], while only first research work can be found in the application and scheduling of mobile manipulators in industrial applica- 
tions, see e.g. [5], [13], [14], [17]. This paper focuses on the development of transport and part-feeding robots as well as path planning and collision avoidance algorithms which are especially adapted to the specific needs of microproduction plants.

Besides the distributed flexible system structure, also the overall automation system consisting of the Manufacturing Execution System (MES) and the shop floor control for a microproduction system has to be designed in a suitable way. Herein, the very flexible and distributed system structure with a possible changing number of involved machines and robots at run-time and the requirements of mass-customization also suggest the application of a distributed automation system. For its realization, multiagent systems have become a widely accepted approach, see e.g. [8], [9], [10], [15], especially for the realization of distributed MES, see e.g. [6], [7], [10], to mention only a few. These distributed solutions are very well suited to automate flexible production scheduling and resource allocation processes. In the presented approach, we therefore also apply a multiagent-based MES which has to be adapted to include the mobile transportation and part-feeding robots.

The paper is organized as follows. In section 2, we propose the overall systems architecture of the flexible microproduction system in more detail, also describing the development of the mobile robots. In section 3, the multiagent-based MES is explained while the special distributed navigation, path planning and collision avoidance algorithms adapted to microproduction are described in section 4 . Finally, developed analysis and 3D-simulation tools as well as simulation results of the overall resulting microproduction system are presented in section 5 .

\section{Overall System Structure}

A microproduction system that fulfils the mentioned requirements must be flexible with regard to the system structure as well as the structure of the automation system, in order to form automated and adaptable sequences of manufacturing processes. In the proposed solution it is assumed that the overall system consists of a number of stationary microproduction and -assembly modules from different suppliers and of different sizes that offer all necessary production and assembly processes. In order to achieve a highly flexible interconnection of these stationary modules, it is assumed that the modules are interconnected via mobile transport robots. These transport robots are able to transport parts and tools on a selfdefined path between the stationary modules and thus provide the highly flexible and self-configuring production processes by interconnecting the production processes in the required sequence, see Fig. 1 for a sketch of the overall system structure.

The application of mobile transport robots in manufacturing or logistics is not new, so called automated guided vehicles (AGVs) are already applied there [4], [12]. How-

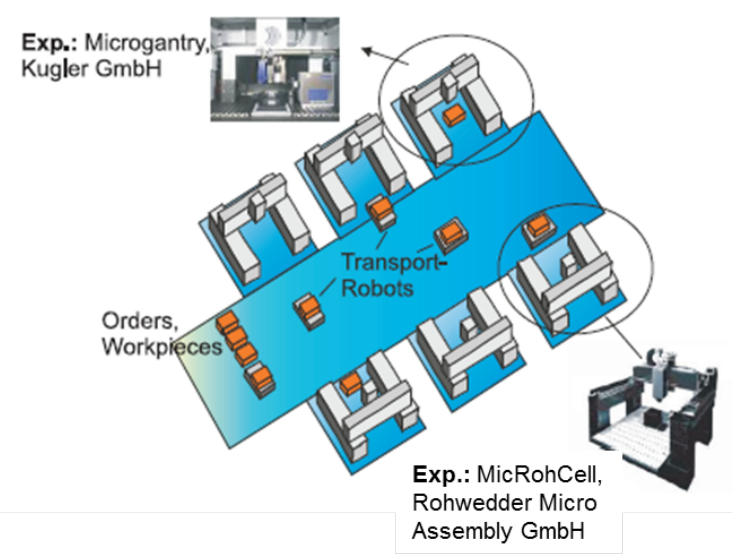

\section{Figure 1. Overall flexible microproduction system structure.}

ever, the AGVs used so far are only automatically following a fixed pre-defined path which is marked by a lane or landmarks. In contrast to these AGVs, the proposed solution here comprises autonomous mobile robots that are freely navigating in a workspace based on own sensor information (laser scanners, cameras etc.) and hereby able to avoid collisions with other robots and human coworkers. However, from a navigational perspective, since the environment with the stationary machines is not completely unknown but can be stored as a map, the mobile robots hereby only have to perform localization instead of the more complex simultaneous localization and mapping (SLAM) task, see e.g. [4]. The main advantages of this free navigation are the advanced flexibility of the robots that are able to connect machines in an arbitrary way, the possibility to easily and rapidly change or extend the overall system and the possibility to easily connect clean rooms (as often required in microproduction processes).

For a highly automated microproduction system however, the robots do not only have to transport parts and tools between the machines but also have to take over the part-feeding task which has to be done with a suitable manipulator. Since the parts and products in microproduction are very small, their automatic handling and also clamping is extremely difficult and a research topic of its own. In order to provide an applicable solution here and to focus on the realization of the overall microproduction system, this problem is solved by fixing the parts on a pallet system. The parts can then be placed or fixed on the pallet in a separate working step while only the pallets are transported and fed to the machines during production. In commercially available pallet solutions, automatic clamping of the pallets with high precision is possible which also relaxes the clamping problem. Finally, the mobile robots have to be equipped with a manipulator that is able to handle the pallets only and feed them to the machines. A prototype of such a robot with a lightweight manipula- 


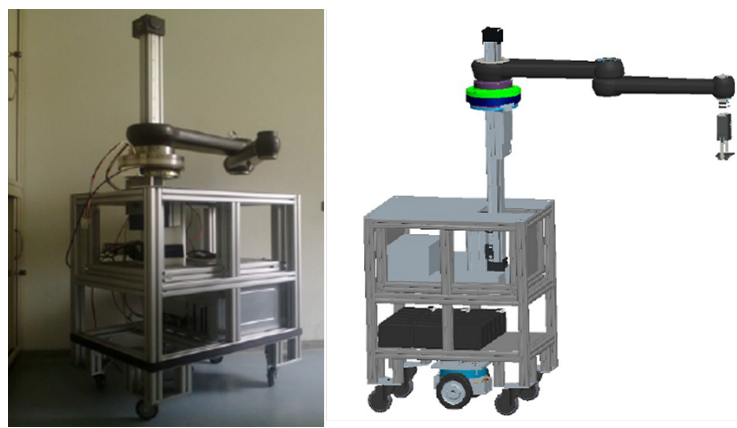

Figure 2. Single mobile transport and partfeeding robot.

tor of suitable range has been developed in this project, see Fig. 2. The control of the manipulator is done based on visual information, see e.g. [17] and will not be considered here in detail since the main focus is on the integration of multiple robots in the overall concept. However, the task of transporting pallet systems with very tiny and sensitive parts also creates constraints that have to be considered during the navigation of the mobile robots, see section 4 .

\section{Multiagent-Based Automation System for Microproduction}

The structure of the overall automation system for the flexible microproduction system is first of all organized referring to the well-known automation pyramid, see Fig. 3, comprising the enterprise resource planning (ERP) layer, the manufacturing exection system (MES) and the field control layer. The main focus in this contribution is on the MES and the field control, where we apply distributed solutions in accordance with the flexible distributed overall system structure as described before. For the realization of distributed solutions, multiagent systems have become a widely accepted approach, see e.g. [8], [9], [10], [15], especially for the realization of distributed MES, see [6], [7], [10], for an overview.

As described in [6], the concept of a MES is defined as a set of functions that cover all aspects of the management of the shop floor and provide communication with the ERP. The MES Association (MESA) and the ISA SP95 define eleven functions that characterize the main functionality of MES and mainly include scheduling and dispatching of orders and resource allocation, data acquisition and collection as well as labour, quality, process and maintenance management. Many solutions for the realization of MES exist, ranging from centralized to completely distributed structures, see also e.g. [6], [7] for an overview. The distributed solutions are mainly based on multiagent systems, which can either represent a functional or structural decomposition of the overall MES.

As many other multiagent-based approaches, also the MES proposed here comprises a set of generally used agents (see Fig. 3): order agents which represent the microproduction orders, transport agents representing the mobile robots with a given transport resource, machine agents representing the microproduction and assembly machines with a given production capability, broker agents that realize the resource allocation as well as supervisory agents (neglected in Fig. 3).

The order agents are responsible for the realization of the production orders where each order is represented by one agent. An order consists of a production request for a required number of a specified microproduct. The order agents have access to a common database which includes the product models of the microproducts that can be produced with the given microproduction system. The order agent negotiates the resource allocation with the machine agents and the transport agents using an auction mechanism that is coordinated by the broker agent. In order to describe the microproducts and the related production orders, a hierarchical product model has been derived, see Fig. 4 for a simple example, which is stored by the order agents.

Herein it is assumed that initial materials are transformed into intermediate and final products with the help of a defined sequence of suitable microproduction process steps. Those transformation sequences are represented as a directed graph with links and nodes, where nodes represent materials, intermediate or final products and links represent interdependencies between them. The start node of a single link represents an element that depends on the element associated to the end node. Furthermore, each link is associated with a number that indicates how many end node elements are necessary in order to generate the start node elements. The start nodes are also indicating the necessary production process that has to be processed on a suitable machine.

The machine agents represent the production or assembly machines included in the microproduction system. The agents are responsible for the execution of produc-

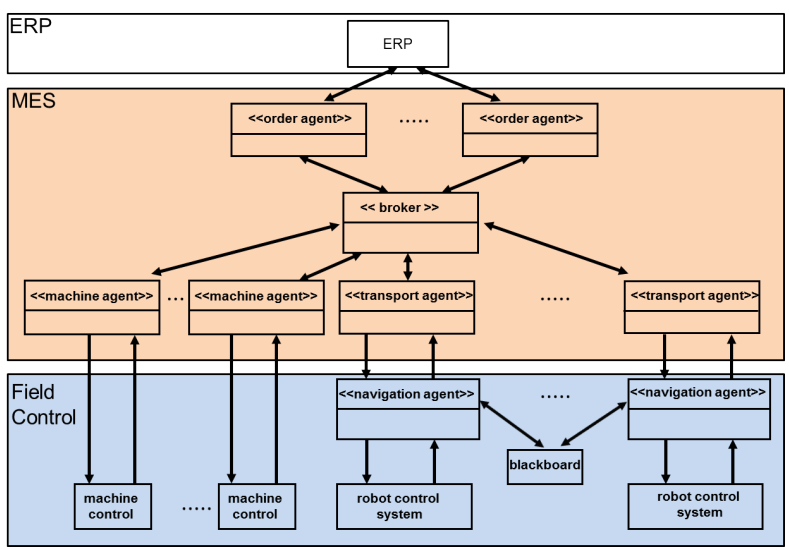

Figure 3. Structure of the overall automation system. 


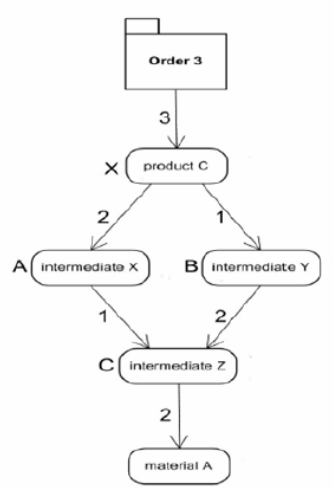

Figure 4. Microsystem product model.

tion processes and are ordered for that purpose by the order agents. Before allocating a machine to an order, the respective machine agent is negotiating with the order agents via the broker agent. Hereby, the machine agent contains the knowledge regarding the production capacity as well as the capabilities of the associated machine.

The transport agents are responsible for the allocation of the transport capacity of the associated transport robots. The agents are negotiating via the broker agent with the order agents in order to achieve transport orders. The transport agents have the knowledge about the transport capacity and the mapping information about the environment. After the assignment of a transport order, they also know the required time schedule (start time and latest arrival time) as well as the start and end position for the transport task. While the transport agents are therefore concerned with the long term planning of the transport tasks, the mid term motion planning and control tasks are taken over by a navigation agent which is associated to each transport agents as described in the following section. These navigation agents are considered as a part of the field control layer as shown in Fig. 3 since they are mainly providing real time control tasks.

The online allocation of the machining and transport capacities to the microproduction orders is solved via an auction mechanism, see e.g. [18], coordinated by a broker agent. First, the order agent determines all required production process steps of its related microproduction order using the product model. The agent then negotiates the required machining and transport capacities and the next allocation schedule. If the negotiation is successful, the microproduct will be generated accordingly, otherwise the order agents wait and start a new negotiation process later. It is assumed that the order agents have a limited budget for the realization of the microproduction orders, their main goal is to maximize their utility, i.e. generation of the microproduct in short time. The machine and transport agents are suppliers that offer their capacities as a service and try to maximize their profits. The final allocation is negotiated in form of a Dutch auction (see e.g. [18]) where the suppliers offer their capacities at the beginning with the highest price and reduce the price with increasing auction time. The Dutch auction has been chosen since it generally leads to a reduced duration of the auction comparing to other auction forms. The order agents are periodically considering the offered capacities and try to buy the required capacities for the next production steps. The broker agent acts first of all as a blackboard where all offers are published, and finally also confirms the successful deal between two participants. In this type of allocation mechanism, the order agent are always buying the capacities that they need for the next steps. If this step is completed, the order agents starts buying the next capacities based on the current situation. This leads to a very flexible distributed allocation that is able to react to disturbances or changing situations, while the overall efficiency might be reduced comparing to a long term centralized planning approach. Regarding a study of the efficiency of different auction types and multiagent based allocation mechanisms we also refer e.g. to [18].

\section{Coordinated Control of the Multi-Robot Transport System}

After the allocation of the transport capacities to the transport agents, the remaining task on the field control layer is the coordinated navigation of the multi-robot system during the transportation tasks using navigation agents (NA). We assume that each NA receives the information about the next transportation task provided by the respective transport agent in the form of start position and time, destination position and latest delivery time as well as the assigned transportation order. The NAs then have to fulfil these transportation tasks in the best possible way while the special microproduction environment adds some problem-specific constraints. Since the space between the stationary machine tools is free (i.e. without further stationary obstacles) but limited, and since several robots always operate in parallel, the biggest problem for the robots is collision avoidance with other moving robots. In addition, since the robots have to transport extremely small work pieces in pallet systems which should not be disordered too much, the accelerations both in and perpendicular to the travel direction as well as velocities and turning rates are limited. In addition, the robots should move energy-efficient in order to increase the operating time with one battery charge.

In this project, we apply an adapted version of prioritized planning on the global long-term level for planning rough collision free paths defined by waypoints for all the robots, see also [4], [17]. This approach fits well to the underlying transportation problem: if any NA starts a transportation task, we generally assume that the already moving robots have a higher priority. Therefore, the considered NA computes its own collision free path with the help of a model predictive approach taking the already determined paths of the other prioritized robots as fixed. In order to do so, the NA have access to a common blackboard where the path information from other NAs are pub- 
lished, see Fig. 3. This approach then has to be extended to include differential constraints. In order to simplify the algorithms, our approach only considers velocity constraints on the global long-term planning level and more detailed differential constraints on the local real-time control level. For global motion planning, the velocities of the robots are considered as being constant but limited between two waypoints, respectively. Planning under differential constraints also has been intensively studied, see e.g. [4]. One useful approach is the discretization of the constraints by using a simplified discrete-time model of the robotic motion. In this work, the result of the global long-term decoupled planning under simplified differential constraints is a priority relation between the robots and a set of collision free waypoints for all robots from the start to the goal location with a fixed limited velocity for each way-segment between two waypoints.

However, it must be taken into account that in reality uncertainties of the used models during planning as well as uncertainties of the measurements and unforeseen events during the execution of the plans can have a strong influence on the overall resulting motion of the robots. Problems of this type are also intensively studied in the literature, see again [4] for an overview, and solutions are e.g. using probabilistic planning or dynamic re-planning on the global long-term level. In this project, those problems are not solved on the global long-term planning level but are combined with the solution of the path following problem and therefore solved on a local, real-time motion control level. Here, we interpret the solution of the long-term motion planning as a set of paths that must be followed by the NAs with a "desired" velocity on the respective path segments. If these conditions are perfectly fulfilled this would result in collision free paths. Because of the mentioned uncertainties however, we cannot generally guarantee that no collisions occur if we only try to realize the long-term motion planning.

Therefore, all NA are continuously combining the task of path following with collision avoidance under detailed differential constraints on the local real-time motion control level. Hereby the main task for each NA is to follow the specified path with the desired velocity while continuously checking for any possible collision. This is done with the help of a blackboard and the knowledge about all current locations of the robots and therefore in a coordinated fashion, see also Fig. 3. The problem of motion control like path following is also investigated in the literature, see e.g. [4] for an overview. One promising approach which motivates the proposed solution is based on model predictive control for the path following or tracking problem since it offers a natural way to include differential constraints. In addition, this contribution extends a non-linear model predictive path following algorithm with collision avoidance to a very efficient overall approach.

The mathematical details of the overall approach are neglected here, we therefore refer to [16], [17]. In the following, we finally show some simulation results of the re-

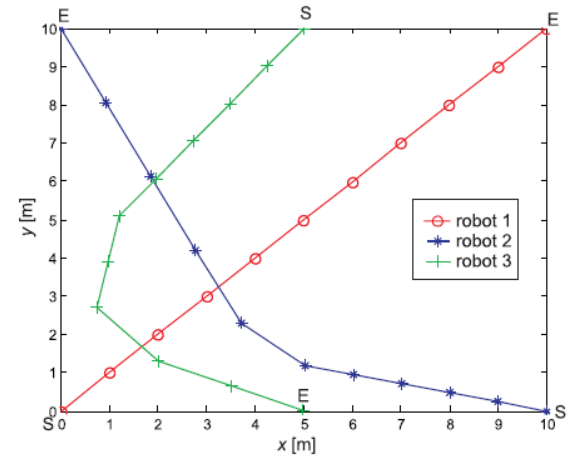

\section{Figure 5. Result of the global long-term mo- tion planning.}

alized multiagent-based distributed navigation approach. In a first simulation which is intended to proof the concept of the global long-term motion planning, three robots are considered in an $\mathrm{x}$-y-coordinate system of the microproduction plant. The robots start at the same time after prioritization where robot 1 has the highest, robot three the lowest priority. The result of the decoupled prioritized planning is shown in Fig. 5. Herein, $\mathrm{S}$ denotes the start and $\mathrm{E}$ the goal location, and the markers denote the calculated waypoints, respectively. It becomes obvious that robot 1 with the highest priority drives on the direct way from start to the goal location, herein keeping the velocity constraints. Robot 2 then has to take this path of robot 1 into account and to plan a path where the distance between these two robots is always larger than three meters. Finally, robot 3 has the lowest priority and to adapt its path to the two other already computed paths of robot 1 and 2 . Also in this case, the obtained path of robot 3 keeps a distance of a least three meters between robot 3 and the other two robots. In all cases, the velocity constraints are also fulfilled.

Finally, also the real-time motion control approach is tested in a simulation. In this experiment two robots 1 and 2 meet and robot 2 has the higher priority. Therefore, robot 2 only has to follow the desired path, as depicted in Fig. 6. Robot 1 then has to follow the path while having an initial deviation from the desired path and always has to keep a distance of a least 0.4 meters from the other robot. The results of the model predictive approach as depicted in Fig. 6 underline its efficiency. Robot 1 first tries to minimize the deviation from the desired path, however then it has to start avoiding the approaching robot 2 . That results in a deviation from the desired path of robot 1 again. After robot 2 has passed, robot 1 is again approaching the desired path. Fig. 6 also shows that the collision avoidance constraints are always fulfilled. The result can be interpreted as the best compromise between path following and collision avoidance while additionally keeping the differential constraints. 


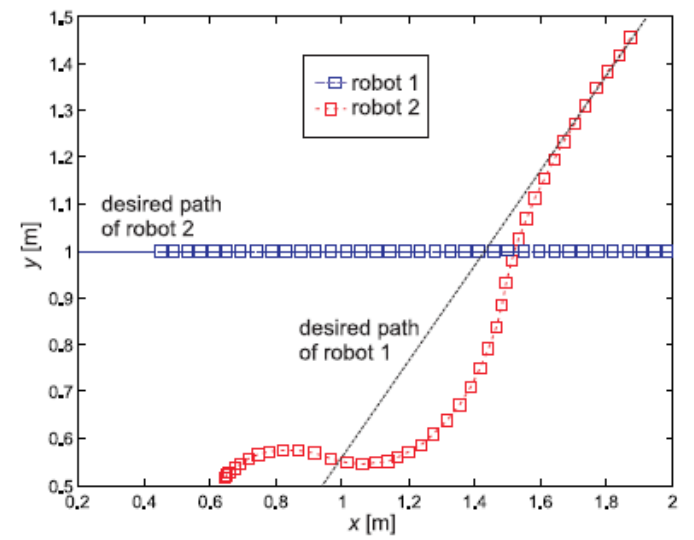

Figure 6. Results of predictive control of the single robots.

\section{Design Tools and Simulation Results}

In order to support the development of a flexible microproduction system in the proposed form, design and support tools have been developed in this project. The multiagent systems for the MES as well as the navigation agents have been developed using the Living Systems Technology Suite LS/TS from Whitestein Technologies (www.whitestein.com). In addition, a tool to design a simulation model of the overall microproduction system also including the multi-robot transport system has been developed using the Webots 3D robot simulation environment from Cyberbotics Ltd. (www.cyberbotics.com). With the help of Webots, a repository of pre-configured microproduction machines as well as transport and part-feeding robots was created. Herein, the mechnical 3D construction of the robots and the manipulators generated with a suitable CAD tool and the dynamic behaviour described by differential equations can be modelled. In addition, also models of sensors like cameras and laser scanners can be generated from the Webot repository and included in the robots. Herein, the signal processing of the sensors is integrated in the robot simulation, while the sensors create real-time sensor data during their movement in the simulated environment. Finally, also a virtual 3D environment can be added to form the overall 3D simulation model of the microproduction system in VRML (Virtual Reality Modeling Language) as shown in Fig. 7. This 3D simulation can furthermore be connected with the multiagent system and the shop floor control systems in order to generate 3D simulations of the overall microproduction processes.

In addition to this simulation tool that supports the model-based design and 3D simulation of the microproduction system, an evaluation tool has been developed in order to analyse the details as well as the overall performance of the multiagent-based automation system. Therefore, a $\log$ file is generated during the runtime of the pre-

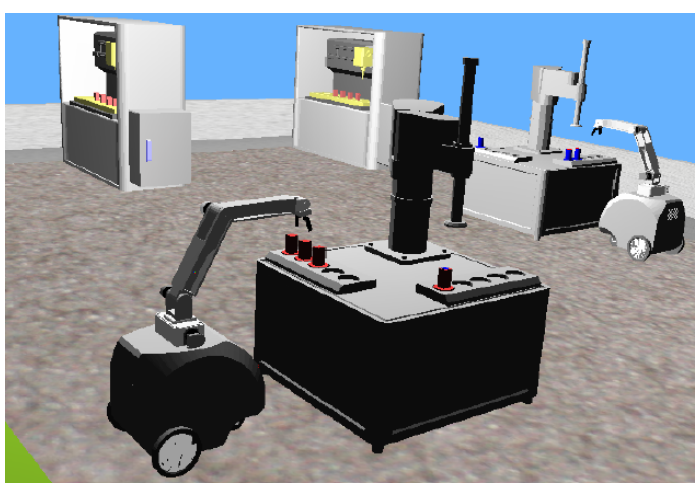

Figure 7. 3D-simulation of the microproduction system.

viously mentioned simulation of the microproduction system, where all events, allocation and scheduling processes are stored. With the help of a web-based graphical user interface, the simulation data in the log file can be transformed into graphics such as Gantt charts to display the details of the resource allocation or the results of the auction mechanism, see e.g. Fig. 8 for an example of such a simulation result.

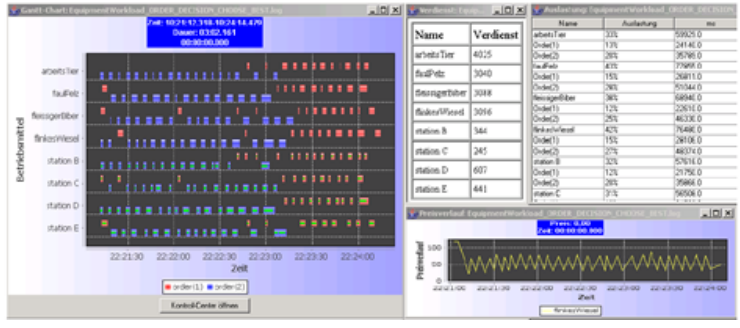

\section{Figure 8. Analysis of the microproduction process.}

In this example, the allocation schedule of the different machines as well as transport robots is shown in the Gantt chart, where each row describes the occupation of the capacities. In addition, the budget distribution and also the decreasing of the budget during the production process is given in graphical form. The graphical visualization of the simulation results allows the designer to analyse the efficiency of the current system configuration and to adapt important parameters such as the budget distribution of the agents in order to optimize the overall system behaviour in an iterative process of design, simulation and adaptation.

\section{Summary}

This contribution proposes a new concept of a flexible microproduction system that provides two main innovations. First, the proposed concept integrates stationary production machines and mobile transport robots in order to configure rapidly changing production processes in 
real-time. Besides this distributed flexible system structure, also the overall automation system consisting of the Manufacturing Execution System (MES) and the shop floor control is designed in a distributed form as a multiagent system. This distributed automation system is especially suited to automate flexible production scheduling and resource allocation processes but also integrates the multi-robot transport system. In addition, suitable simulation and analysis tools have been developed to support the design of such a microproduction system, first simulation results underline the applicability of the proposed approach.

\section{References}

[1] S. Büttgenbach, A. Burisch, J. Hesselbach (Eds.). Design and Manufacturing of Active Microsystems. Berlin: Springer, 1. Edition, 2011.

[2] H. Weule, J. Fleischer, J. Elsner. Investigation on the International State of the Art of Micro Production Technology. In: Proc. of European Society for precision engineering and nanotechnology (euspen), May 19-20, 2003, Aachen (D), p. 11-18.

[3] A. Hofmann, et al. Evolvable Micro Production Systems: Specific needs and differences to macro. In: Proc. of the IEEE Int. Symposium on Assembly and Manufacturing (ISAM), 2011, pp. 1-6.

[4] B. Siciliano, O. Khatib. Springer Handbook of Robotics. Berlin: Springer, 2008.

[5] O. Brock, C.C. Kemp. Guest editorial: special issue on autonomous mobile manipulation. In Autonomous Robots, Springer, vol. 28, no. 1, January 2010.

[6] A. Bratukhin, T. Sauter. Functional Analysis of Manufacturing Execution System Distribution. In IEEE Trans. on Industrial Informatics, vol. 7, no. 4, pp. 740 - 749, Nov 2011.

[7] A. Bratukhin, T. Sauter. Bridging the Gap between Centralized and Distributed Manufacturing Execution Planning. In: Proceedings of 15th IEEE International Conference on Emerging Techonologies and Factory Automation (ETFA 2010); Bilbao, 2010, S. $1-8$.

[8] R. Unland, et al. Industrial Agent Technology. In: Wilamowski, Bogdan M.; Irwin, J. David (Ed.), The Industrial Electronics Handbook - Industrial Communication Systems, 2. ed.; Boca Raton, FL: CRC Press, 2011, S. 16-1 - 16-15.

[9] W. Lepuschitz, A. Zoitl, M. Valle, M. Merdan. Towards Self-Reconfiguration of Manufacturing Systems Using Automation Agents. In: IEEE Trans. on Systems, Man, and Cybernetics, Part C: Applications and Reviews, vol.41, no.1, pp.52-69, Jan. 2011.
[10] A. Lüder, A. Klostermeyer, J. Peschke, A. Bratoukhine, T. Sauter. Distributed automation: PABADIS versus HMS. In: IEEE Trans. on Industrial Informatics, vol.1, no.1, pp. 31-38, Feb. 2005.

[11] S. Datta, R. Ray, D. Banerji. Development of autonomous mobile robot with manipulator for manufacturing environment. In: The International Journal of Advanced Manufacturing Technology, Springer, August 2008, Volume 38, Issue 5-6, pp 536-542.

[12] B. Horan, et al. OzTug mobile robot for manufacturing transportation. In: Conference proceeding of the 2011 International Conference on Systems, Man, and Cybernetics, SMC 2011, Anchorage, USA, pp. 3554-3560.

[13] V.Q. Dang, et al. Scheduling a Single Mobile Robot for Part-Feeding Tasks of Production Lines. In: Journal of Intelligent Manufacturing, 2013.

[14] V.Q. Dang, I. Ewa Nielsen. Multiple mobile robots scheduling problem in industrial production environment. In: Proc. International Symposium on Management Intelligent Systems. 2013.

[15] H. Voos. Agent-based Distributed Resource Allocation in Continuous Dynamic Systems. In: Ahmed, S.; Karsiti, M. (Eds): Multiagent Systems, I-Tech, Vienna, 2009, pp. 1-19.

[16] H. Voos. Model Predictive Collaborative Motion Planning and Control of Mobile Robots including Safety Aspects. In: Proc. of the Int. Conference on Advanced Robotics ICAR 2009, Munich, 2009.

[17] S. Wangmanaopituk, H. Voos, W. Kongprawechnon. Collaborative Nonlinear Model-Predictive Motion Planning and Control of Mobile Transport Robots for a Highly Flexible Production System. In: ScienceAsia, 36 (2010) 4, pp. 333-341.

[18] G. Weiss (edt.). Multiagent Systems. Cambridge (MA): MIT press, 2. edition, 2013. 